\title{
Safety and efficacy of pralatrexate in the treatment of patients with relapsed or refractory peripheral T-cell lymphoma
}

\author{
Enrica Marchi and Owen A. O'Connor
}

\begin{abstract}
T-cell lymphomas (TCL) are a diverse and heterogeneous group of malignancies that represent less than $15 \%$ of all non-Hodgkin lymphomas. Initial refinements of the clinical classification of these complex diseases have been made, but a better understanding of their molecular pathogenesis is still needed. Even if the paucity of insights into the underlying pathogenesis of TCLs has hindered our ability to develop rational targeted therapies, significant advances have been made. Pralatrexate (10-propargyl 10-deazaaminopterin) is a unique antifolate that has been rationally designed to have high affinity for the reduced folate receptor (RFC) and the folylpolyglutamate synthetase (FPGS) and was the first drug ever approved for the treatment of relapsed and refractory peripheral T-cell lymphomas (PTCL). This review describes the preclinical development of pralatrexate that led to early-phase clinical trials in lung cancer and lymphoma and its subsequent approval in PTCL. The review also describes how pralatrexate has been combined with other agents in both the preclinical and clinical settings. FDA approval for the use of pralatrexate in PTCL has been granted based on the results of the pivotal Phase II trial of this agent in relapsed and refractory PTCL patients.
\end{abstract}

Keywords: clinical development, pralatrexate, preclinical data, T-cell lymphoma

\section{Introduction}

Peripheral T-cell lymphoma (PTCL) is a collective term to designate malignancies derived from the post-thymic mature $\mathrm{T}$ cells and natural killer (NK) cells. PTCLs encompass a heterogeneous group of diseases, altogether accounting for less than $15 \%$ of all non-Hodgkin lymphomas (NHLs) worldwide [Vose et al. 2008]. The World Health Organization (WHO) classification [World Health Organization, 2008], which is based on a combination of morphologic, immunophenotypic, genetic and clinical features, as well as correlation with a normal cellular counterpart, has been used since 2008. While a major advance over other classification schema, its application has been hampered by a paucity of cytogenetic and molecular markers. PTCLs are typically grouped according to their presentation as: (1) disseminated; (2) predominantly extranodal; (3) cutaneous or (4) predominantly nodal (Table 1 ). It has long been recognized that the majority of PTCLs have an inferior response to therapy and a worse prognosis compared with their B-cell counterparts.
Treatment approaches have mirrored those implemented for diffuse large B-cell lymphoma. As a result, CHOP (cyclophosphamide, doxorubicin, vincristine and prednisone) and CHOPlike regimens containing etoposide have been considered 'the standard therapy' despite consistently disappointing results. The dismal outcome of patients with PTCL has created a substantial need to develop more efficacious treatment options. The first of several drugs recently approved for patients with PTCL was pralatrexate, which received accelerated approval from the US Food and Drug Administration (FDA) with the name of Folotyn for the treatment of relapsed or refractory PTCLs in September 2009.

\section{Pharmacology}

Like other antifolates, pralatrexate inhibits the folate metabolism pathway through inhibition of dihydrofolate reductase (DHFR). DHFR converts dihydrofolate, a reduced form of dietary folate, to tetrahydrofolate, which is used in the
Ther Adv Hematol

(2012) 3(4) 227-235

DOI: $10.1177 /$

2040620712445330

(c) The Author(s), 2012. Reprints and permissions: http://www.sagepub.co.uk/ journalsPermissions.nav
Correspondence to: Enrica Marchi, MD, PhD Associate Research Scientist, Center for Lymphoid Malignancies, Department of Medicine, Columbia University Medical Center, New York, NY 10032, USA em2517acolumbia.edu

Owen A. O'Connor, MD, PhD

Center for Lymphoid Malignancies, Department of Medicine, Columbia University Medical Center, New York, NY, USA 
Table 1. WHO Classification, 2008

\begin{tabular}{l} 
WHO Classification 2008 \\
\hline Precursor T-cell lymphoma \\
T-Lymphoblastic Lymphoma/leukemia \\
Mature T-cell lymphomas \\
T-cell prolymphocytic leukemia \\
T-cell large granular lymphocytic leukemia \\
Aggressive NK-cell leukemia \\
Indolent large granular NK-cell \\
lymphoproliferative disorder (provisional) \\
ATL/adult T-cell leukemia \\
Extranodal NK-/T-cell lymphoma, nasal type \\
Enteropathy-associated T-cell lymphoma (EATL) \\
Hepatosplenic T-cell lymphoma \\
Subcutaneous panniculitis-like T-cell lymphoma \\
l $\alpha \beta$ only) \\
Primary cutaneous $\gamma \delta$ T-cell lymphoma \\
Mycosis Fungoides/Sézary syndrome \\
Anaplastic large-cell lymphoma (ALCL) ALK+ \\
ACLC ALK- (provisional) \\
PTCL NOS \\
Angioimmunoblastic T-cell lymphoma (AITL) \\
Primary cutaneous CD30+ T-cell LPD \\
LYP and primary cutaneous ALCL \\
Primary cutaneous CD4+ aggressivesmall/ \\
medium T-cell lymphoma (provisional) \\
Primary cutaneous CD8+ aggressive \\
epidermotropic cytotoxic T-cell lymphoma \\
(provisional) \\
Systemic EBV+ T-cell LPD of childhood \\
Hydroa vaccinoforme-like lymphoma \\
\hline
\end{tabular}

synthesis and catabolism of several amino acids, the formation of creatine and choline, the synthesis of purines, the methylation of ribonucleic acids and the synthesis of thymidine monophosphate (TMP). The metabolic inhibition of DHFR by pralatrexate results in depletion of TMP and other precursors essential in DNA and RNA synthesis, therefore halting cellular proliferation.

Pralatrexate (10-propargyl-10-deazaaminopterin) is the prototype of a new class of antifolates belonging to the class of molecules known as 10-deazaaminopterin (Figure 1(a)). These compounds are structurally designed to have a higher affinity for the reduced folate carrier (RFC) and for the folypolyglutamyl synthase (FPGS) leading to enhanced intracellular uptake and accumulation in the tumor cells [Sirotnak et al. 1982, 1984]. Pralatrexate enters cancer cells via RFC, after which it is polyglutamated by FPGS in the cytosol (Figure 1(b)). Compared with methotrexate, pralatrexate is more efficiently internalized and retained in the cancer cell. The $K_{m}$ values for
RFC for pralatrexate and methotrexate are 0.3 and $4.8 \mu \mathrm{mol} / 1$, respectively, whereas the $V_{\max } / K_{m}$ values (rate of intracellular transport) are 12.6 and 0.9 , respectively. These data establish that the rate of pralatrexate influx is nearly 14 -fold greater than for methotrexate. Following a similar pattern, the $K_{m}$ values for pralatrexate and methotrexate for FPGS are 5.9 and $32.3 \mu \mathrm{mol} / 1$, respectively, whereas the $V_{\max } / K_{m}$ for folypolyglutamyl synthase is 23.2 and 2.2 , respectively. These biochemical data likewise suggest a greater potential for pralatrexate to be both internalized and retained inside tumor cells expressing RFC compared with other traditional antifolates.

\section{Preclinical study}

\section{Pralatrexate as a single agent}

Pralatrexate was originally developed by Sirotnak and colleagues at Memorial Sloan Kettering in collaboration with Southern Research Institute International. Initial in vitro studies in the NCI cancer cell panel demonstrated that pralatrexate was potently cytotoxic across a broad panel of cancer cell types, including solid tumors and hematologic malignancies. The efficacy of pralatrexate was compared with methotrexate, an antifolate that has been used for a very long time in the treatment of aggressive NHL. The activity of pralatrexate was compared with methotrexate against five lymphoma cell lines: RL (transformed follicular lymphoma), HT, SKI-DLBCL-1 (diffuse large B cell), Raji (Burkitt's), and Hs445 (Hodgkin's disease). Pralatrexate demonstrated more than 10-fold greater cytotoxicity than methotrexate in all cell lines $\left(\mathrm{IC}_{50}\right.$ pralatrexate $=$ 3-5 nM, IC 50 methotrexate $=30-50 \mathrm{nM})$. The activity of pralatrexate and methotrexate was also compared in vivo against three established NHL xenograft NOD/SCID mice (HT, RL, and SKI cells were injected). Across the different lymphoma xenograft models pralatrexate demonstrated statistically superior tumor growth inhibition compared with methotrexate. These results reported that pralatrexate demonstrated markedly greater in vitro and in vivo activity against NHLs than methotrexate and warranted further preclinical and clinical evaluation [Wang et al. 2003]. Recently the activity of pralatrexate has been investigated in different multiple myeloma (MM) lines. Pralatrexate induced concentration-dependent apoptotic cell death in a subset of human myeloma cell lines (HMCL) and $\mathrm{CD}_{138^{+}} \mathrm{MM}$ cells isolated from a clinical 


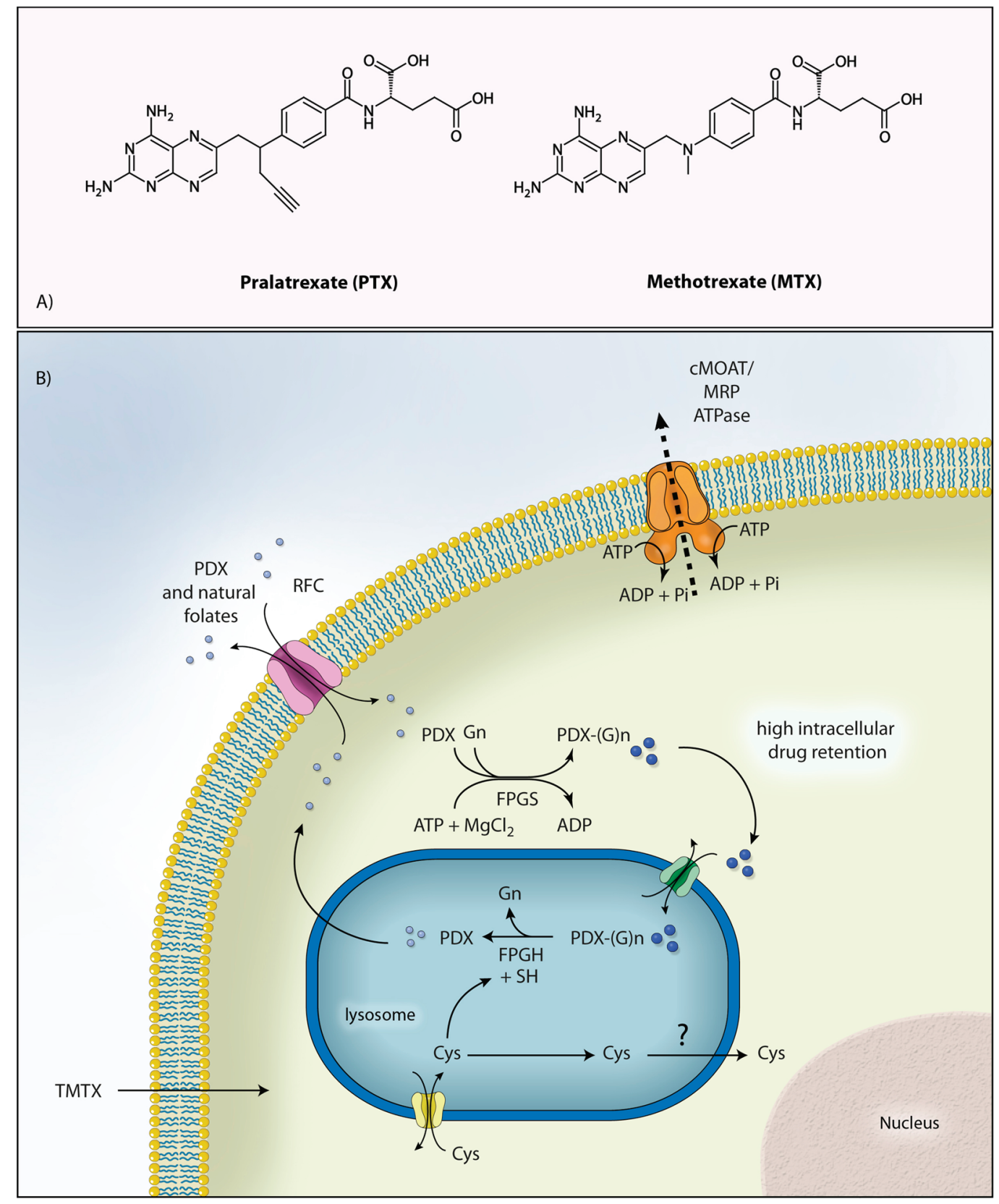

Figure 1. Figure 1. A: Chemical structure of pralatrexate and methotrexate; B: Internalization and retention of pralatrexate inside the cells. PDX, pralatrexate; RFC, reduced folate carrier; PDX-(G)n, polyglutamated pralatrexate; TMTX, trimetrexate; FPGS, folylpolyglutamate synthase; FPGH, folypolyglutamyl hydrolase; cMOAT, canalicular multispecific organic-anion transporter; MRP, multidrug resistance-associated protein. Illustration courtesy of Alessandro Baliani. Copyright (C) 2012. Adapted from Owen O'Connor's personal slide.

specimen. In sensitive cell lines, pralatrexate exhibited 10-fold greater potency compared with methotrexate. Pralatrexate induced concentration-dependent intrinsic apoptosis in sensitive HMCLs, characterized by cleavage of caspase- 3 and -9 and accompanied by the loss of full-length Mcl-1, a Bcl-2 family protein that plays a critical role in drug-induced apoptosis in MM. The authors showed that the activity of pralatrexate was not abrogated by the presence of exogenous interleukin- 6 or by coculture with HS- 5 bone marrow stromal cells, both of which exert powerful survival effects on MM cells and can antagonize apoptosis in response to some cytotoxic 
chemotherapy drugs. Sensitivity to pralatrexateinduced apoptosis correlated with higher relative levels of RFC mRNA in sensitive compared to resistant HMCL. Resistant HMCL exhibited a concentration-dependent up-regulation of dihydrofolate reductase (DHFR) protein in response to pralatrexate exposure, whereas sensitive HMCL did not. Interestingly, these changes in functional folate metabolism biomarkers, high baseline RFC expression and upregulation of DHFR in response to pralatrexate, appeared to be mutually exclusive in sensitive or resistant HMCL (i.e. in sensitive cell lines, elevated RFC and poor upregulation of DHFR; in resistant cell lines, low RFC and robust upregulation of DHFR), respectively. In addition, pralatrexate was also effective against sensitive HMCL in vivo in a novel mouse xenograft model (NOG mice inoculated with MM.1s HMCL stably transduced to express both GFP and luciferase [GFP-luc]). Treatment with pralatrexate resulted in a significant reduction in tumor burden after two doses. These data supported further investigation of pralatrexate in preclinical and early clinical model of MM [Mangone et al. 2011].

\section{Pralatrexate used in combination}

Pralatrexate has been evaluated in preclinical combination studies. It is already known that methotrexate synergizes with cytarabine (1-h-D-arabinofuranosylcytosine [ara-C]) in a schedule-dependent manner. Toner and colleagues [Toner et al. 2006] compared the activity of pralatrexate plus gemcitabine with the standard combination of methotrexate plus ara-C. The study demonstrated that the combination of pralatrexate followed by gemcitabine was superior to methotrexate/ara-C in the in vitro and in vivo models and was far more potent in inducing apoptosis in DLBCL. To further evaluate the activity of pralatrexate in combination with other T-cell active drug [Zinzani et al. 2007], our group investigated the activity of the combination of pralatrexate with the proteosome inhibitor, bortezomib [Marchi et al. 2010]. In vitro, pralatrexate and bortezomib exhibited concentration and time-dependent cytotoxicity against a broad panel of T-lymphoma cell lines. The combination of pralatrexate and bortezomib synergistically induced apoptosis and caspase activation across the panel of T-cell lymphoma lines studied (two cutaneous T-cell lymphomas [CTCL] lines, $\mathrm{H} 9$ and $\mathrm{HH}$ and two T-ALL lines, P12 and PF382). Studies on normal peripheral blood mononuclear cells (PBMCs) showed that the combination of pralatrexate and bortezomib was not more toxic than the single agents. Western blot assays for proteins involved in broad growth and survival pathways demonstrated that $\mathrm{p} 27$, NOXA, HH3, and RFC were all significantly modulated by the combination. In a SCID beige mouse model of transformed CTCL, the addition of pralatrexate to bortezomib enhanced efficacy compared with either drug alone. Collectively, these data suggested that pralatrexate in combination with bortezomib represents a novel and potentially important platform for the treatment of T-cell malignancies [Marchi et al. 2010]. A phase I/II clinical trial in now underway based on this preclinical data.

\section{Early clinical experience}

The first phase I study of this agent was carried out in patients with relapsed or refractory nonsmall-cell lung cancer (NSCLCA) who had received a median of two prior therapies [Krug et al. 2000]. Initially the drug was administered at the dose of $30 \mathrm{mg} / \mathrm{m}^{2}$ weekly for 3 weeks on a 4-week cycle. Mucositis requiring dose interruption or dose reduction occurred in four out of six patients. The treatment schedule was then modified to every 2 weeks and 27 patients were treated starting at $15 \mathrm{mg} / \mathrm{m}^{2}$ with the dose being escalated to $170 \mathrm{mg} / \mathrm{m}^{2}$. On this schedule, the recommended dose was $150 \mathrm{mg} / \mathrm{m}^{2}$ given on alternate weeks on days 1 and 15 of a 28-day cycle. Two of 33 patients on this trial experienced an objective response including a complete remission (CR) and 5 patients had stable disease. This study was then extended into a phase II trial at a dose of 150 $\mathrm{mg} / \mathrm{m}^{2}$ every 2 weeks in patients with stage III/IV lung cancer who were previously untreated or had progressed after initial therapy [Krug et al. 2003]. A total of 39 patients were enrolled of which 38 were evaluable. Four patients had an objective response lasting $4,9,12$, and 15 months, while 12 patients experienced stable disease. The median time to progression was 3 months and the predicted 1 - and 2-year survival rates were $56 \%$ (43-74\%) and 36\% (25-58\%) respectively.

Other strategies to further improve the efficacy of pralatrexate included combining it with probenecid [Fury et al. 2006]. The combination strategy was supported by preclinical in vivo data demonstrating that the combination of pralatrexate and probenecid could significantly enhance the antitumor effect of pralatrexate in different models of human mesothelioma. A phase I trial of 17 patients 
was performed with a combination of pralatrexate and probenecid to assess the maximum tolerated dose (MTD) of this combination. The combination could be delivered but there were no antitumor responses seen. Pralatrexate was studied in combination in a variety of other studies including a phase I clinical trial in which pralatrexate was used with paclitaxol or docetaxol in patients with NSCLCA and other solid tumors [Azzoli et al. 2007]. An MTD for the combination was not established but the MTD for pralatrexate was reached at $120 \mathrm{mg} / \mathrm{m}^{2}$ when combined with docetaxol. There were 6 partial response and 20 patients had stable disease out of the 40 patients with NSCLCA treated. A phase II trial of pralatrexate in mesothelioma was carried out at a pralatrexate dose of $135 \mathrm{mg} / \mathrm{m}^{2}$ given every 2 weeks [Krug et al. 2007]. Of the 16 patients that were treated on this protocol, there were no responses.

\section{Clinical development in hematological malignancies}

Methotrexate is an active drug in many subtypes of lymphoma and is an integral part of some combination chemotherapy regimens that are used in the treatment of these diseases at various dose levels. As described above, preclinical data indicate that pralatrexate is more potent than methotrexate. This has led investigators to study the activity of this agent in patients with lymphomas and the initiation of the first dedicated trial in lymphomas. A phase I trial was opened using pralatrexate at the dose of $135 \mathrm{mg} / \mathrm{m}^{2}$. This dose level was selected based upon the MTD defined in lung cancer patients. Pralatrexate was given every other week (QOW) in patients with relapsed/ refractory Hodgkin's lymphoma and NHL with the purpose of determining the MTD and efficacy of pralatrexate in patients with lymphoma [O'Connor et al. 2009]. Dose escalation was allowed by $15 \mathrm{mg} / \mathrm{m}^{2}$ if no toxicity was observed after two cycles. A total of 16 patients were treated including five patients with Hodgkin's disease, eight patients with aggressive lymphomas, two patients with mantle cell lymphoma, and one patient with PTCL. All patients experienced stomatitis at this dosing schedule with $44 \%$ grade 3 and $6 \%$ grade 4 stomatitis. Other grade 3 or 4 events included leukopenia (62\%), lymphopenia $(69 \%)$, and thrombocytopenia (52\%). The study revealed that the incidence of stomatitis was associated with elevated level of homocysteine and methylmalonic (6 out of 16 patients with grade III/ IV mucositis) whereas no mucositis was noted in patients with homocysteine and methylmalonic acid levels less than $10 \mu \mathrm{mol} / 1$ and $200 \mathrm{nM} / \mathrm{l}$, respectively. The supplementation of folic acid and vitamin B12 prevented mucositis in many of these patients [Mould et al. 2009]. In addition, the idiosyncratic area under the curve (AUC) of exposure was found to be the second determinant of toxicity. Patients with high AUC exposures to pralatrexate experienced substantially higher risk of mucositis compared with those with lower AUC exposures. Between the nutritional and PK covariates, virtually $100 \%$ of the risk of mucositis could be predicted based on these determinants alone. Interestingly, the only patient who experienced a complete response was a man with a history of chemotherapy refractory PTCL NOS documented by a negative CT scan and PET scan following a single dose of pralatrexate. Owing to the high incidence of stomatitis, the study was amended to a weekly phase I dose-escalation study based on laboratory data suggesting superior efficacy of a weekly schedule with less toxicity. The dose escalation was conducted as follows: 30 $\mathrm{mg} / \mathrm{m}^{2}$ weekly $\times 3$ every 4 weeks; $30 \mathrm{mg} / \mathrm{m}^{2}$ weekly $\times 6$ every 7 weeks; then increasing by $15 \mathrm{mg} / \mathrm{m}^{2}$ on the 7 -week schedule. All patients received supplementation with vitamin B12 and folic acid. A total of 17 patients with both NHL and Hodgkin's disease were enrolled in this QOW study and the maximum administered dose (MAD) of $45 \mathrm{mg} / \mathrm{m}^{2}$ was established. At this dose the cycle 1 dose limiting toxicities included neutropenic fever, neutropenia, and thrombocytopenia. In the overall cohort the incidence of stomatitis was $17 \%$, pharyngitis $4 \%$, and leukopenia $30 \%$. This was a marked reduction in toxicity compared with the QOW schedule. There were two deaths in the 45 $\mathrm{mg} / \mathrm{m}^{2}$ cohort thought to be secondary to the underlying disease and compromise of anatomical barriers that led to life-threatening infections. There were no other toxicities in the expanded cohort and $45 \mathrm{mg} / \mathrm{m}^{2}$ dose was declared the MAD. The MTD was determined to be $30 \mathrm{mg} / \mathrm{m}^{2}$ given weekly for 6 out of 7 weeks and studied further in another 24 patients in the phase II portion of the study with response rate as an endpoint. Overall 48 patients were treated on this trial with a wide range of lymphoma diagnoses. The overall response rate (ORR) was $31 \%$ with CR in $17 \%$ of patients. The majority of the responses were seen in patients with a T-cell lineage lymphoma including PTCL NOS, anaplastic large cell lymphoma (ALCL), and angioimmunoblastic T-cell lymphoma (AITL) (four of the first five patients had T-cell lymphoma and they achieved a CR within 
Table 2. Clinical trials of pralatrexate in hematologic malignancies

\begin{tabular}{|c|c|c|c|c|}
\hline TRIAL & DOSE & PATIENTS & TOXICITY & RESPONSE \\
\hline PDX 02-78 & $\begin{array}{l}135 \mathrm{mg} / \mathrm{m}^{2} \text { Q2 weeks } \\
30 \mathrm{mg} / \mathrm{m}^{2} \text { weekly x } 3 \text { Q4 } \\
\text { weeks + Vitamin B12 / folate } \\
30 \mathrm{mg} / \mathrm{m}^{2} \text { weekly x } 6 \text { every } 7 \\
\text { weeks + Vitamin B12 / folate }\end{array}$ & $\begin{array}{l}\text { NHL relapsed } \\
N=48\end{array}$ & $\begin{array}{l}\text { Mucositis grade } 3 \\
(44 \%) \\
\text { Mucositis grade } 3 \\
(17 \%)\end{array}$ & $\begin{array}{l}\text { ORR } 31 \% \\
\text { CR } 17 \%\end{array}$ \\
\hline $\begin{array}{l}\text { PDX-008 } \\
\text { (PROPEL) }\end{array}$ & $\begin{array}{l}30 \mathrm{mg} / \mathrm{m}^{2} \text { weekly x } 6 \text { Q } 7 \\
\text { weeks + Vitamin B12 / folate }\end{array}$ & $\begin{array}{l}\text { PTCL relapsed } \\
N=115\end{array}$ & $\begin{array}{l}\text { Mucositis, } \\
\text { thrombocytopenia }\end{array}$ & $\begin{array}{l}\text { ORR 27\% } \\
\text { (Pivotal Trial) }\end{array}$ \\
\hline PDX-010 & $\begin{array}{l}30,20,15,10 \mathrm{mg} / \mathrm{m}^{2} \text { weekly x } \\
3 \text { every } 4 \text { weeks (or) } \\
\text { Weekly x } 2 \text { every } 3 \text { weeks + } \\
\text { vitamin B12 and folate }\end{array}$ & $\begin{array}{l}\text { CTCL relapsed } \\
N=31\end{array}$ & $\begin{array}{l}\text { Mucositis grade } 3 \\
(13 \%), \text { fatigue and } \\
\text { thrombocytopenia }\end{array}$ & $\begin{array}{l}\text { MTD }=15 \mathrm{mg} / \mathrm{m}^{2} \\
\text { weekly } \times 3 \\
\text { ORR } 56 \% \text { (dose } \geq \\
15 \mathrm{mg} / \mathrm{m}^{2} \text { ) }\end{array}$ \\
\hline PDX-009 & $\begin{array}{l}\text { Gemcitabine + pralatrexate } \\
\text { dose escalation - weekly x } 3 Q \\
4 \text { weeks and } Q 2 \text { weeks } \\
\text { Same-day administration } \\
\text { Sequential-day } \\
\text { administration }\end{array}$ & $\begin{array}{l}\text { NHL relapsed } \\
N=33\end{array}$ & $\begin{array}{l}\text { Fever, } \\
\text { thrombocytopenia, } \\
\text { mucositis, } \\
\text { neutropenia, } \\
\text { pulmonary embolus }\end{array}$ & $\begin{array}{l}\text { MTD } 15 \mathrm{mg} / \mathrm{m}^{2} \text { and } \\
600 \mathrm{mg} / \mathrm{m}^{2} \text { (same } \\
\text { day) } \\
\text { MTD } 10 \mathrm{mg} / \mathrm{m}^{2} \\
\text { and } 400 \mathrm{mg} / \mathrm{m}^{2} \\
\text { (sequential day) }\end{array}$ \\
\hline
\end{tabular}

the first cycle of treatment). The ORR was $10 \%$ in patients with B-cell lymphoma and 54\% in patients with T-cell lymphomas. All patients with a CR had a diagnosis of $\mathrm{T}$ cell NHL while $4 / 6$ with a partial response (PR) were PET negative. The duration of response was 3-26 months. This trial established the specific class activity of pralatrexate in T-cell lymphomas at the dose level of $30 \mathrm{mg} / \mathrm{m}^{2}$ given as a single agent on a 6-out-of-7week schedule. This observation has led to the pivotal trial of this agent in relapsed/refractory PTCL and its recent approval for this indication. Normalization of homocysteine and methylmalonic levels prior to therapy with folic acid and vitamin B12 supplementation abrogated the mucositis. Hematological toxicity was minimal and only grade 2 thrombocytopenia was noted in two patients. This study established that there was a significant specific activity of pralatrexate in patients with T-cell lymphomas and warranted a confirmatory phase II study.

The multicenter PROPEL study (Pralatrexate in Relapsed or Refractory Peripheral T-cell Lymphoma) [O'Connor et al. 2011] was developed under the FDA Special Protocol Assessment (SPA) program. Pralatrexate was given US and EU orphan drug designation for T-cell NHL and an FDA fast track designation for approval in September 2009. The study was opened at multiple US and International cancer centers to ensure rapid accrual. All histologies of T-cell NHL were eligible for the study including transformed mycosis fungoides, HTLV-1 adult T-cell leukemia/ lymphoma (HTLV-1 ATLL) and the rare forms of NK T-cell lymphomas. Patients had to have measurable disease and had to have progressed on at least one prior treatment with no restriction on the prior number of therapies as long as they had adequate hematological, hepatic, and renal function. The primary endpoint was response rate (RR) as defined by the IWC criteria with duration of response, progression-free survival (PFS) and overall survival (OS) as secondary endpoints. The study required independent central review of pathology and response along with an independent data monitoring committee that performed three independent safety assessments. A total of 115 patients were enrolled between August 2006 and April 2008, of which 109 were evaluable. In general, this was a very heavily pretreated population with a median of $3(1-12)$ prior treatment regimens including 18 patients with a prior autologous transplant. Interestingly, $20 \%$ of patients had received more than five lines of prior therapy. A total of $53 \%$ of the patients were refractory to the last regimen, while $25 \%$ of the patients never had a response to any therapy indicating primary refractory disease. The treatment schedule consisted of pralatrexate given at $30 \mathrm{mg} / \mathrm{m}^{2}$ weekly for 6 weeks followed by 1 week of rest on a 7 -week cycle. Folic acid and vitamin B12 supplementation was carried out in all patients. Based on an independent review, an overall response rate of $29 \%$ was noted in all patients, with nine patients $(12 \%)$ achieving CR/CRu. A total of $69 \%$ of the 
responses occurred after cycle 1 of therapy. There were 14 IWC+PET responses based on the revised Cheson criteria published in 2007 [Cheson et al. 2007]. This trial reported a median duration of response of 10.4 months. Further analysis of the data has shown that even patients who had more than two prior regimens of therapy including prior autologous stem cell transplantation had a response rate of $30 \%$ which in a heavily pretreated population is impressive. Four responding patients went on to definitive therapy with stem cell transplant. Mucosal inflammation was seen in over $70 \%$ of patients but was grade 3 and 4 in only $21 \%$ of patients. Hematologic toxicity consisted of thrombocytopenia, which was grade 3 in $14 \%$ and grade 4 in $19 \%$ of cases. A grade 3 anemia was seen in only $16 \%$ of case. Other toxicities included mild fatigue, nausea, dyspnea, and mild abnormalities of LFTS and serum electrolytes. Febrile neutropenia was noted in only $5 \%$ of cases. This was the largest prospective study ever conducted for relapsed or refractory PTCL patients and showed very promising activity of pralatrexate in this disease despite being studied in a very heavily treated patient population. The PROPEL trial led to the FDA approval of pralatrexate for the treatment of relapsed and refractory T-cell lymphoma. Table 2 lists the clinical trials of pralatrexate in hematologic malignancies.

\section{Clinical development in CTCL}

CTCLs are a heterogeneous group of T-cell lymphoproliferative disorders involving the skin, the majority of which may be classified as Mycosis fungoides (MF) or Sézary syndrome (SS). Given the specific T-cell class activity of pralatrexate [O'Connor et al. 2009] and the generally indolent course of this disease, a phase 1 clinical dosereduction trial in patients with relapsed or refractory CTCL was initiated. The primary objective was to identify the optimal dose and schedule of pralatrexate for these patients. In the initial pralatrexate phase I study in lymphoma, impressive responses were seen in patients with CTCL along with other T-cell malignancies at a MTD of 30 $\mathrm{mg} / \mathrm{m}^{2}$. Owing to the indolent nature of the disease, a dose de-escalation study of pralatrexate was designed in CTCL with the intent of finding the least-toxic dose with activity in this population. Eligible patients with a diagnosis of CTCL including SS or cutaneous anaplastic large cell lymphoma were eligible if they had failed at least one prior systemic therapy. There were two dosing schema with a 3 -out-of-4-week schedule and a 2-out-of-3-week schedule. Doses were to be reduced in a sequential cohort based on toxicity with optimal dose and schedule defined as evidence of antitumor activity assessed by the modified severity weighted assessment tool (mSWAT) without grade 4 hematological toxicity, grade 3-4 infection or febrile neutropenia. A total of 31 patients received pralatrexate. Patients had received a median of 6 (range, 1-25) prior therapies. Patients received pralatrexate for a median of 72 (range, 7-491+) days; four patients received $>10$ cycles of treatment. The most common treatment-related adverse events (all grades) were mucositis $(58 \%)$, nausea $(45 \%)$, fatigue $(45 \%)$, pyrexia $(23 \%)$, vomiting (19\%), anemia (19\%), and edema (16\%). Grade 3-4 treatment-related toxicities in more than one patient each were mucositis (four patients [13\%]) and anemia (two patients [6\%]). Mucositis was dose limiting (grade 2) in eight patients (26\%). A total of 11 responses were observed, including 2 complete responses and 9 partial responses. In the 18 patients who received pralatrexate at a dose intensity of $15 \mathrm{mg} / \mathrm{m}^{2} \times 3 / 4$ weeks or greater, the objective response rate was $56 \%$ (10/18 patients). The results of this trial showed that pralatrexate has high activity with acceptable toxicity in patients with relapsed or refractory CTCL at the identified optimal dose and schedule of $15 \mathrm{mg} / \mathrm{m}^{2}$ weekly for $3 / 4$ weeks. The lack of significant hematologic toxicity or cumulative toxicity suggested that pralatrexate should be further evaluated as continuous or maintenance therapy for patients with CTCL [Horwitz et al. 2012].

\section{Clinical trials using pralatrexate in combination}

Based on the preclinical data that has been discussed above [Toner et al. 2006], a phase I/II trial of the combination of pralatrexate followed by gemcitabine was initiated in patients with lymphoma and Hodgkin's disease [Horwitz et al. 2009]. The primary endpoint was to find the MTD of this combination and to study this dose in a phase II trial to establish efficacy. Three different treatment schedules are being studied with a starting dose of pralatrexate at $10 \mathrm{mg} / \mathrm{m}^{2}$ and gemcitabine at $300 \mathrm{mg} / \mathrm{m}^{2}$. After initial analysis, it has been demonstrated that a weekly schedule is too toxic in pretreated patients with hematologic toxicity being the DLT. The 14-day cycle is 
better tolerated and dose escalation is continuing on this portion of the trial. Preliminary data shows that partial responses were seen across a variety of NHL histologies including patients who had failed previous therapy (data to provide). This demonstrates the feasibility of this combination that has been studied in the lab and which appears to be active in the clinical setting as well at doses that are far below established. In addition, preclinical studies suggested that pralatrexate is synergistic with bexarotene in CTCL lines. A dose-finding, phase I study is now ongoing using pralatrexate in combination with bexarotene in patients with relapsed and refractory CTCL. Preliminary results identified the MTD as $15 \mathrm{mg} / \mathrm{m}^{2}$ pralatrexate $+150 \mathrm{mg} / \mathrm{m}^{2}$ bexarotene. Evaluation of response in a cohort of 30 patients is still ongoing [Duvic et al. 2012].

\section{Future direction}

Despite significant improvements in their clinical and pathologic classification, T-cell lymphomas remain a challenge for all clinicians. Some advances have been made in understanding the molecular pathogenesis of these diseases but the paucity of molecular markers and the lack of knowledge into their pathogenesis have hampered our ability to develop rational targeted therapies for these diseases. However, enormous advances have been made in recent times with the development of several novel classes of drugs, including novel antifolate and histone deacetylase (HDAC) inhibitors, which appear to have relatively unique and reproducible activity in the T-cell lymphomas. Combination studies in both the preclinical and clinical setting have already established that pralatrexate synergizes with a number of other T-cell active agents including gemcitabine, bortezomib, and HDAC inhibitors. These observations have been already translated in phase I/II clinical trials that will certainly provide regarding the most effective and safe strategy to improve patient's outcome.

\section{Funding}

This research received no specific grant from any funding agency in the public, commercial, or notfor-profit sectors.

\section{Conflict of interest statement}

Owen A. O'Connor sits on the scientific advisory board for Allos Therapeutics and receives grant support for research with pralatrexate.

\section{References}

Azzoli, C.G., Krug, L.M., Gomez, J., Miller, V.A., Kris, M.G., Ginsberg, M.S. et al. (2007) A phase 1 study of pralatrexate in combination with paclitaxel or docetaxel in patients with advanced solid tumors. Clin Cancer Res 13: 2692-2698.

Cheson, B.D., Pfistner, B., Juweid, M.E., Gascoyne, R.D., Specht, L., Horning, S.J. et al. (2007) Revised response criteria for malignant lymphoma. $\mathcal{F}$ Clin Oncol 25: 579-586.

Duvic, M., Zinzani, P.L., Geskin, L., Kim, Y.H. and Chance, L. (2012) Pralatrexate plus bexarotene in patients with relapsed or refractory cutaneous $\mathrm{T}$-cell lymphoma (CTCL): study design and preliminary results from an ongoing, open-label, phase 1 dosefinding study. In T-cell Lymphoma Forum, San Francisco, CA, 2012.

Fury, M.G., Krug, L.M., Azzoli, C.G., Sharma, S., Kemeny, N., Wu, N. et al. (2006) A phase I clinical pharmacologic study of pralatrexate in combination with probenecid in adults with advanced solid tumors. Cancer Chemother Pharmacol 57: 671-677.

Horwitz, S.M., Kim, Y.H., Foss, F., Zain, J.M., Myskowski, P.L., Lechowicz, M.J. et al. (2012) Identification of an active, well-tolerated dose of pralatrexate in patients with relapsed or refractory cutaneous T-cell lymphoma (CTCL). Blood, in press.

Horwitz, S.M., Vose, J.M., Advani, R., Sankhala, K., Padmanabhan, S., Hamlin, P.A., Jr et al. (2009) Pralatrexate and gemcitabine in patients with relapsed or refractory lymphoproliferative malignancies: phase 1 results. Blood ASH Annual Meeting 114: abstract 1678.

Krug, L.M., Azzoli, C.G., Kris, M.G., Miller, V.A., Khokhar, N.Z., Tong, W. et al. (2003) 10-propargyl10-deazaaminopterin: an antifolate with activity in patients with previously treated non-small cell lung cancer. Clin Cancer Res 9: 2072-2078.

Krug, L.M., Heelan, R.T., Kris, M.G., Venkatraman, E. and Sirotnak, F.M. (2007) Phase II trial of pralatrexate (10-propargyl-10-deazaaminopterin, PDX) in patients with unresectable malignant pleural mesothelioma. f Thorac Oncol 2: 317-320.

Krug, L.M., Ng, K.K., Kris, M.G., Miller, V.A., Tong, W., Heelan, R.T. et al. (2000) Phase I and pharmacokinetic study of 10-propargyl-10deazaaminopterin, a new antifolate. Clin Cancer Res 6: 3493-3498.

Mangone, M., Scotto, L., Marchi, E., O'Connor, O.A. and Cho, H.J. (2011) Pralatrexate has potent activity against multiple myeloma in vitro and in vivo, and activity correlates with tumor RFC-1 and DHFR expression. ASH Annual Meeting Abstracts 118: abstract 1831 . 
Marchi, E., Paoluzzi, L., Scotto, L., Seshan, V.E., Zain, J.M., Zinzani, P.L. et al. (2010) Pralatrexate is synergistic with the proteasome inhibitor bortezomib in in vitro and in vivo models of T-cell lymphoid malignancies. Clin Cancer Res 16: 3648-3658.

Mould, D.R., Sweeney, K., Duffull, S.B., Neylon, E., Hamlin, P., Horwitz, S. et al. (2009) A population pharmacokinetic and pharmacodynamic evaluation of pralatrexate in patients with relapsed or refractory non-Hodgkin's or Hodgkin's lymphoma. Clin Pharmacol Ther 86: 190-196.

O’Connor, O.A., Horwitz, S., Hamlin, P., Portlock, C., Moskowitz, C.H., Sarasohn, D. et al. (2009) Phase II-I-II study of two different doses and schedules of pralatrexate, a high-affinity substrate for the reduced folate carrier, in patients with relapsed or refractory lymphoma reveals marked activity in T-cell malignancies. F Clin Oncol 27: 4357-4364.

O’Connor, O.A., Pro, B., Pinter-Brown, L., Bartlett, N., Popplewell, L., Coiffier, B. et al. (2011) Pralatrexate in patients with relapsed or refractory peripheral T-cell lymphoma: results from the pivotal PROPEL study. F Clin Oncol 29: 1182-1189.

Sirotnak, F.M., DeGraw, J.I., Chello, P.L., Moccio, D.M. and Dorick, D.M. (1982) Biochemical and pharmacologic properties of a new folate analog, 10-deaza-aminopterin, in mice. Cancer Treat Rep 66: 351-358.

Sirotnak, F.M., DeGraw, J.I., Moccio, D.M., Samuels, L.L. and Goutas, L.J. (1984) New folate analogs of the 10-deaza-aminopterin series. Basis for structural design and biochemical and pharmacologic properties. Cancer Chemother Pharmacol 12: 18-25.

Toner, L.E., Vrhovac, R., Smith, E.A., Gardner, J., Heaney, M., Gonen, M. et al. (2006) The schedule-dependent effects of the novel antifolate pralatrexate and gemcitabine are superior to methotrexate and cytarabine in models of human non-Hodgkin's lymphoma. Clin Cancer Res 12: 924-932.

Vose, J., Armitage, J. and Weisenburger, D. (2008) International peripheral $\mathrm{T}$-cell and natural killer/Tcell lymphoma study: pathology findings and clinical outcomes. F Clin Oncol 26: 4124-4130.

Wang, E.S., O'Connor, O., She, Y., Zelenetz, A.D., Sirotnak, F.M. and Moore, M.A. (2003) Activity of a novel anti-folate (PDX, 10-propargyl 10-deazaaminopterin) against human lymphoma is superior to methotrexate and correlates with tumor RFC-1 gene expression. Leuk Lymphoma 44: 1027-1035.

World Health Organization (2008) WHO Classification of Tumours of Haematopoietic and Lymphoid Tissues (4th edn). Lyon: IARC Press.

Zinzani, P.L., Musuraca, G., Tani, M., Stefoni, V., Marchi, E., Fina, M. et al. (2007) Phase II trial of proteasome inhibitor bortezomib in patients with relapsed or refractory cutaneous T-cell lymphoma. f Clin Oncol 25: 4293-4297.
Visit SAGE journals online http://tah.sagepub.com

(SAGE journals 